\title{
Altered expression of the CSMD1 gene in the peripheral blood of schizophrenia patients
}

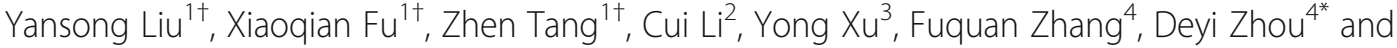 \\ Chunming Zhu $^{4^{*}}$ (D)
}

\begin{abstract}
Background: Schizophrenia (SCZ) is a heritable, refractory, and devastating psychiatric disorder. Previous studies have shown that the variants of CUB and sushi multiple domains 1 (CSMD1) demonstrate significant genome-wide association with SCZ. However, few studies have been conducted on the effect of antipsychotics on the expression levels of CSMD1. This study explored whether a change occurs in the expression of the CSMD1 gene before and after antipsychotic treatment in SCZ patients.
\end{abstract}

Methods: The study population comprised Han Chinese patients from eastern China, including 32 SCZ patients and 48 healthy controls. The expression of CSMD1 before and after treatment in the SCZ group and between the two groups was analyzed using real-time quantitative polymerase chain reaction (RT-qPCR).

Results: The expression levels of the CSMD1 gene in the peripheral blood mononuclear cells (PBMCS) of SCZ patients were lower than those in the healthy controls. The expression levels of the CSMD1 gene in the PBMCs of the SCZ patients after antipsychotic treatment were higher than those in the baseline SCZ patients (all $P<0.05$ ).

Conclusions: Our results showed that the expression levels of CSMD1 are correlated with the development and treatment of SCZ, providing further evidence for the involvement of CSMD1 in SCZ.

Keywords: Schizophrenia, CSMD1, Antipsychotics

\section{Background}

Schizophrenia (SCZ) is a hereditary, disabling, and common psychiatric disorder with a worldwide prevalence of approximately $1 \%$. The typical symptoms of SCZ usually appear in youth, with chronic deregulation of thoughts, perceptions, emotions, and behaviors. Genetic factors are the most crucial mechanism in the etiology of SCZ, accounting for approximately $80 \%$; however, these genetic mechanisms remain unclear $[1,2]$.

The CUB and sushi multiple domains 1 (CSMD1) gene is a complement control-related protein located on $8 \mathrm{p} 23.2$, the variants of which were reported to have a strong correlation with SCZ risk in a recent study [3].

\footnotetext{
* Correspondence: 397556051@qq.com; 13358119677@189.cn;

a126fuxiaoqian@126.com

†Yansong Liu, Xiaoqian Fu and Zhen Tang contributed equally to this work. ${ }^{4}$ Department of Clinical Psychology, Wuxi Mental Health Center, Nanjing Medical University, 156 Qianrong Road, Wuxi, Jiangsu Province 214151, China Full list of author information is available at the end of the article
}

The single-nucleotide polymorphism (SNP) rs10503253 inside the CSMD1 gene has been reported to be a major genome-wide risk locus for SCZ [4], and has also been shown to be associated with multiple neurodevelopmental disorders $[3,5,6]$. Studies on SCZ patients of European ancestry have shown that the A risk allele of rs10503253 is related to impaired cognitive function in SCZ $[4,7]$ and it has been verified as a risk allele of SCZ [8-10]. Moreover, the disruption of CSMD1 may be related to neuropsychological deficits in csmd1 knockout mice [11]. Furthermore, CSMD1 has been reported as being involved in regulating the ratio between dopamine and serotonin metabolites in cerebrospinal fluid [12]. Collectively, these findings indicate that the CSMD1 gene is closely related to the neurogenesis, cognition, immunity, neuropsychology, and monoamine metabolism of SCZ. However, recent studies on the relationship between CSMD1 and SCZ have been inconsistent;

(c) The Author(s). 2019 Open Access This article is distributed under the terms of the Creative Commons Attribution 4.0 International License (http://creativecommons.org/licenses/by/4.0/), which permits unrestricted use, distribution, and reproduction in any medium, provided you give appropriate credit to the original author(s) and the source, provide a link to the Creative Commons license, and indicate if changes were made. The Creative Commons Public Domain Dedication waiver (http://creativecommons.org/publicdomain/zero/1.0/) applies to the data made available in this article, unless otherwise stated. 
studies have also found no association between rs10503253 and SCZ in patients of Japanese [13] and Han Chinese descent [14].

Although many studies have been conducted on the correlation between CSMD1 and SCZ, few studies have been conducted on variations in the expression of the CSMD1 gene. Changes in gene expression may explain the molecular mechanism of SCZ and the efficacy of antipsychotics against it [15]. The alteration of gene expression can be tested in peripheral blood mononuclear cells (PBMCs) because it can be collected from patients more easily than brain tissue. It also helps to identify the characteristics of the clinical subtypes and prognosis of the disease and possible reactions to medication [16]. More importantly, some studies have found that gene expression in the blood and brain overlap considerably [17] and PBMCs have been used in many gene expression analysis studies, as summarized in a recent review [18].

Changes in some gene expressions have been found to be associated with antipsychotic medication for SCZ. Some studies have found that antipsychotic drugs can change the gene expression related to the metabolic pathway in patients with SCZ $[19,20]$. Several studies have shown that the expression of some genes is overexpressed, whereas that of others is down-regulated before treatment in SCZ patients, and the use of antipsychotics can partially restore the expression to control levels. Studies have also suggested that these genes are associated with positive symptoms of SCZ based on nearly $95 \%$ of patients having a favorable response to drugs $[16,20]$. This may indicate that some of the beneficial effects of antipsychotic drugs are mediated by regulation of the expression of these genes.

Although many other gene expression changes have been found to be related to the efficacy of antipsychotic drugs, studies on the effects of antipsychotics on the expression of CSMD1 in SCZ are largely absent from the worldwide literature. The CSMD1 gene is closely related to SCZ, and the aforementioned studies indicated that changes in its expression are involved in the molecular mechanism of SCZ and the efficacy of antipsychotics against it. Therefore, whether CSMD1 gene expression is affected by antipsychotic drug treatment warrants investigation. In this study, we aimed to determine the expression level of the CSMD1 gene in PBMCs before and after antipsychotic treatment to explore the therapeutic value of CSMD1 in SCZ.

\section{Methods}

\section{Patient recruitment}

We recruited $32 \mathrm{SCZ}$ patients (17 females and 15 males) and 48 healthy controls (HCs) (31 females and 17 males), all of whom were from a Han Chinese population in
Shanxi Province, China. No obvious statistical differences were observed between the SCZ group and the HC group in terms of sex, age, or race (Table 1).

The case group comprised SCZ patients recruited from the First Hospital of Shanxi Medical University who conformed to the criteria of the Diagnostic and Statistical Manual of Mental Disorders, Fifth Edition (DSM-V), as confirmed by two experienced psychiatrists. Patients with SCZ were not treated with antipsychotic medication before they entered the group. A total of 32 SCZ patients were treated with oral second generation antipsychotics (risperidone $(n=5)$, olanzapine $(n=10)$, quetiapine $(n=6)$, ziprasidone $(n=2)$, aripiprazole $(n=6)$, amisulpride $(n=3))$ and were followed-up for 12 weeks. Based on the evaluation of a Positive and Negative Syndrome Scale reduction rate of over $25 \%$, the clinical symptoms of the patients in the case group were all improved. The exclusion criteria included severe organic brain injury, mental retardation, epilepsy, alcohol or substance abuse, and other mental disorders.

The HC participants were randomly recruited from local communities in Shanxi Province, and they had no mental or neurological diseases according to a Structured Clinical Interview for DSM-IV and Non-patients. The exclusion criteria included those with a family history of mental illness among first-degree relatives, and those born outside Shanxi Province according to a self-assessment questionnaire. The $\mathrm{HC}$ participants and the SCZ patients lived in the same geographic location and were matched for age, sex, and race.

This clinical study was approved by the Ethics Committee of the Wuxi Mental Health Center and the Ethics Committee of the First Hospital of Shanxi Medical University. Informed consent forms were signed by the patients, or by their guardians if they were unable to make their own decisions.

\section{RNA extraction and analysis of gene expression}

A 3-mL sample of peripheral blood was taken from each of the $48 \mathrm{HCs}$ and $32 \mathrm{SCZ}$ patients before and after antipsychotic treatment. Leukocytes were isolated from the fresh blood sample through centrifugation. We extracted total RNA from PBMCs by using TRIzol reagent (Invitrogen, Waltham, MA, USA) with on-column DNase I treatment, as described by the manufacturer. The purity and integrity of total RNA were evaluated using

Table 1 Demographics of HCs and SCZ patients

\begin{tabular}{lllcc}
\hline Variable & HCs & SCZ & t/X2 & $P$ value \\
\hline Sex (M/F) & $17 / 31$ & $15 / 17$ & 1.05 & 0.36 \\
Age (years) & $34.06 \pm 8.04$ & $37.94 \pm 10.30$ & -1.89 & 0.16 \\
Ethnicity & Han & Han & & \\
\hline
\end{tabular}

Notes: HCs: healthy controls; SCZ: schizophrenia patients before treatment; M: male; F: female 
ultraviolet spectrometric measurements and denaturing agarose gel electrophoresis, respectively. A High-Capacity RNA-to-cDNA Kit (Invitrogen) was used to synthesize cDNA, as described by the manufacturer. The expression levels of CSMD1 in the PBMCs of the 48 $\mathrm{HCs}$ and $32 \mathrm{SCZ}$ patients before and after 12-week antipsychotic treatment were measured using real-time quantitative polymerase chain reaction (RT-qPCR) [21], which was performed using a $\mathrm{SYBR}^{\oplus}$ Select Master Mix (Invitrogen). PCR was performed using a 7900HT real-time PCR machine (Applied Biosystems, Foster City, CA, USA) for $2 \mathrm{~min}$ at $50^{\circ} \mathrm{C}$ and $2 \mathrm{~min}$ at $95^{\circ} \mathrm{C}$, followed by 40 cycles consisting of $15 \mathrm{~s}$ at $95^{\circ} \mathrm{C}, 60 \mathrm{~s}$ at $60^{\circ} \mathrm{C}$. Finally, a standard dissociation protocol was used to ensure that each amplicon was a single product. Each RT-qPCR was performed in triplicate for each of the three independent samples. The expression of the glyceraldehyde-3-phosphate dehydrogenase (GAPDH) gene was used as an internal control and all quantifications were normalized to it. The PCR primers for CSMD1 were GTCTGGGCTCGTGGATATGT (forward) and CAGGTCTCGGAAGGACAGAG (reverse).

\section{Statistical analysis}

We used the Statistical Package for the Social Sciences 20.0 for statistical analysis. Sex and age were compared between the SCZ and control groups by using the $x^{2}$ test and an independent-samples $t$ test, respectively. The relative expression level of CSMD1 of each patient after normalization to the glyceraldehyde 3-phosphate dehydrogenase gene was analyzed using the comparative Ct $\left(2^{-\Delta \Delta C t}\right)$ method. The expression levels of CSMD1 were compared between the $\mathrm{HC}$ and SCZ groups by using the Mann-Whitney $U$ test, and the expression of CSMD1 was also compared before and after treatment in the SCZ group by using the Mann-Whitney U test. Two-tailed $P$ values of less than 0.05 were considered to be statistically significant.

\section{Results}

The relative expression levels of the CSMD1 gene in the SCZ patients before treatment, SCZ patients after the 12-week treatment, and the HCs were compared. The results revealed that the expression levels of the CSMD1 gene in the PBMCs of the SCZ patients were lower than in the $\mathrm{HCs}(\mathrm{Z}=-7.54, P<0.01)$. However, after 12 weeks antipsychotic treatment, the expression levels of the CSMD1 gene in the PBMCs of the SCZ patients were markedly increased $(\mathrm{Z}=-6.88, \mathrm{P}<0.01$ ) (Table 2 and Fig. 1).

\section{Discussion}

To the best of our knowledge, this is the first study on changes in the level of CSMD1 expression in SCZ patients
Table 2 Comparison of median CSMD1 levels in SCZ, SCZ_12W, and $\mathrm{HCs}$

\begin{tabular}{llll}
\hline Group & Median $(\mathrm{IQR})$ & Z values & $P$ value \\
\hline HCs $(n=48)$ & $2.51(1.82-6.38)$ & & \\
SCZ $(n=32)$ & $0.05(0.03-0.08)$ & -7.540 & $<0.01$ \\
SCZ_12W $(n=32)$ & $15.75(9.40-35.01)$ & -6.880 & $<0.01$
\end{tabular}

Notes: HCs: healthy controls; SCZ: schizophrenia patients before treatment; SCZ_12w: schizophrenia patients after 12-weeks of treatment; IQR: interquartile range

(1) HC vs SCZ; (2) SCZ vs SCZ_12w

before and after treatment. This study revealed that the expression level of the CSMD1 gene in PBMCs was up-regulated after antipsychotic treatment (Fig. 1 and Table 2). One mouse study that knocked out the CSMD1 gene revealed that it may be associated with the internal phenotypes of mental illness, including partial negative symptoms and a dull emotional response in SCZ [11]. Based on the down-regulated expression of CSMD1 in the $\mathrm{SCZ}$ group compared with the $\mathrm{HC}$ group and the response of CSMD1 levels to antipsychotic drugs, we speculate that disruption of the expression of the CSMD1 gene increases the risk of SCZ. The up-regulation of CSMD1 was consistent with the improvement of mental symptoms in the SCZ patients after antipsychotic treatment, demonstrating that the change of CSMD1 expression was attributable to drug therapy. Consequently, our results indicated that the CSMD1 gene may be a potential biological marker for the treatment of SCZ. Because effects on gene expression are associated with the type of antipsychotic drug [22],

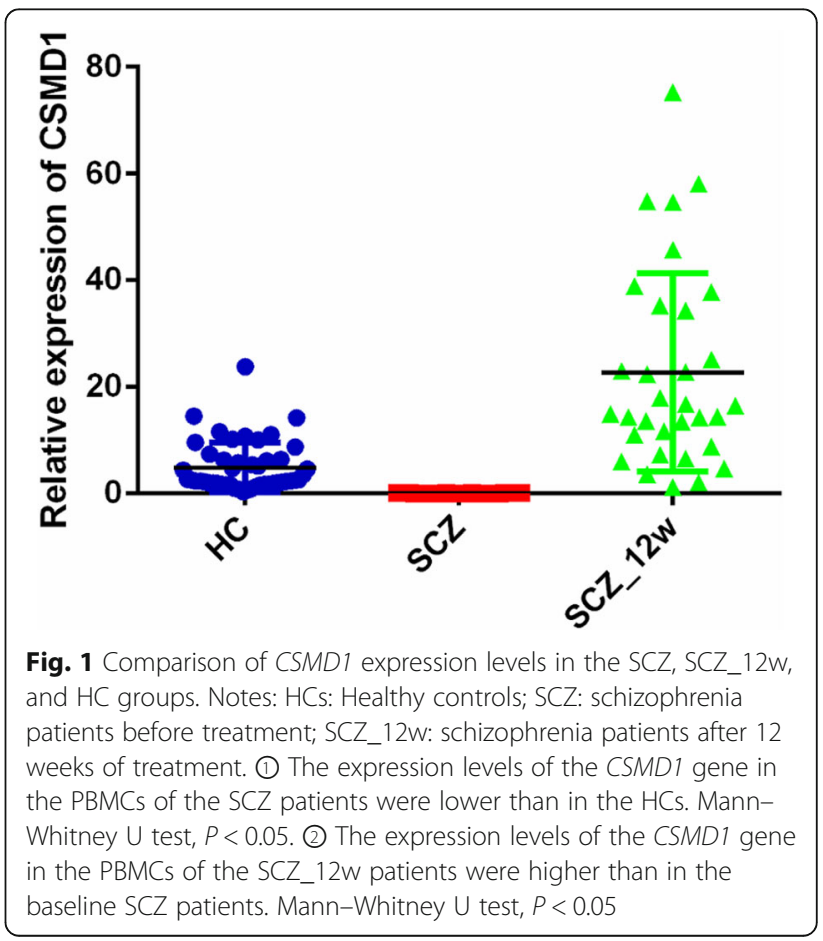


changes in CSMD1 expression may be related to the type of antipsychotic drugs, and their specific relationships warrant further study.

A genome-wide association study [12] on the level of monoamine metabolites in human cerebrospinal fluid revealed that CSMD1 is related to the regulation of the ratio between dopamine and serotonin metabolites in cerebrospinal fluid. More specifically, the risk allele A of CSMD1 is associated with a higher rate of dopamine turnover, which is consistent with the high dopamine state of the etiological hypothesis of SCZ [12]. Another study on the molecular mechanism of antipsychotics revealed that many genes can adjust dopamine receptor levels, indicating that changes in gene expression are consistent with known molecular mechanisms of the antipsychotic effect [20]. The dopamine hypothesis plays a critical role in the etiology of SCZ. Most antipsychotics exert an antagonistic effect on dopamine receptors, which may improve psychotic symptoms by blocking the function of dopamine receptors [23, 24]. This study revealed that the CSMD1 gene had a favorable response to antipsychotic drug intervention, suggesting that CSMD1 may be a new dopamine-related gene involved in the molecular mechanism of antipsychotic drugs.

A previous study was conducted to determine the gene expression that can predict the efficacy of antipsychotic drugs, revealing that genes associated with SCZ are generally of higher predictive value [25]. The CSMD1 gene, the variants of which are strongly correlated with SCZ risk [3], plays a role in preventing complement $\mathrm{C} 3$ activation $[26,27]$. Studies have indicated that complement components (including complement C3) help the brain develop and function by making precise connections to the synapses and neurons [28, 29]. Abnormality of a complement may affect the risk of neurodegenerative and mental illness by causing abnormal synaptic elimination in the brain [30]. In addition, CSMD1 has been reported to be the target of MIR137, which has a strong association with SCZ [3] and is involved in the regulation of adult neurogenic and neuronal maturity [31]. In summary, previous studies have shown that CSMD1 is closely related to SCZ. Our results showed that the $C S M D 1$ gene has a favorable therapeutic response to antipsychotic drugs, thus proving that CSMD1 may be a candidate gene for predicting the therapeutic response to antipsychotic drugs.

The CSMD1 gene is related to immune function through complement regulation, but the precise mechanism remains unclear [26]. The CSMD1 gene is related to complement regulation and encodes a protein related to complement control through the replication of multiple CUB and sushi domains [11]. CSMD1 may also play a critical role in regulating complement activation and inflammation in the developing central nervous system [26, 27]. Notably, antipsychotic drugs such as phenothiazine can affect immune-related genes, leading to oxidative stress in the surrounding tissues [22]. For instance, serum C-reactive protein is substantially increased in patients taking typical antipsychotic drugs [22]. However, the exact mechanism of the drug effect on the immunological aspects of CSMD1 needs further study.

Our research has some limitations. First, the patient population was relatively small, which may have affected the statistical effect in comparing the CSMD1 gene expression level between the two groups. In the future, larger sample sizes are needed to validate our findings. Second, considering the effects of inflammation or antipsychotic drugs, the same gene may be expressed differently in different cell types. Hence, more accurate cell typing is necessary to study gene expression in the future. Third, a caveat to interpretation of results was that we used only one control gene (GAPDH) for normalization in the RT-qPCR experiments, so the results may be influenced by changes in GAPDH.

\section{Conclusions}

Our results indicated that the expression of CSMD1 is associated with both the development and antipsychotic treatment of SCZ, suggesting that the CSMD1 gene may be a promising biological marker for SCZ.

\section{Abbreviations}

CSMD1: CUB and sushi multiple domains 1: DSM-IV: Diagnostic and Statistical Manual of Mental Disorders, 4th edition; F: Female; GAPDH: Glyceraldehyde3-phosphate dehydrogenase; GWAS: Genome-wide association study;

HCS: Healthy controls; IQR: Interquartile range; M: Male; RT-qPCR: Real-time quantitative polymerase chain reaction; SCZ: Schizophrenia;

SCZ: Schizophrenia patients before medication; SCZ_12w: schizophrenia patients after 12-week medication; SNP: Single-nucleotide polymorphism

\section{Acknowledgments}

The authors thank all participants for their cooperation in our study.

\section{Funding}

This study was funded by grants from the Primary Research \& Development Plan of Jiangsu Province (BE2016630), the Suzhou Municipal Bureau of Science and Technology Program (SYS201578, SYSD2017140), Key Research Program of the Suzhou municipal health and Family Planning Commission (LCZX201719, LCZX201615).The funding body had no role in the study design; in collection, analysis, and interpretation of data; and in writing the manuscript.

Availability of data and materials

For access to the data in this paper, interested researchers may contact the corresponding author via email: 13358119677@189.cn.

\section{Authors' contributions}

$C Z$, DZ designed the study. YL, XF, FZ, ZT, YX CL performed the data collection and $Y L, F Z, Z T, Y X$ performed data analyses. $Y L$ and $X F$ were responsible for manuscript writing. All authors read and approved the final manuscript.

Ethics approval and consent to participate

This study was approved by the Ethics Committees of the Wuxi Health Mental Center. All participants signed a written informed consent prior to participation in the study. 


\section{Consent for publication}

Not applicable.

\section{Competing interests}

The authors declare no conflicts of interest.

\section{Publisher's Note}

Springer Nature remains neutral with regard to jurisdictional claims in published maps and institutional affiliations.

\section{Author details}

'Department of Clinical Psychology, Suzhou Guangji Hospital, The Affiliated Guangji Hospital of Soochow University, Suzhou 215137, Jiangsu, China. 2Department of Psychology, Xinghua People's Hospital, Xinghua 225700, Jiangsu, China. ${ }^{3}$ Department of Psychiatry, First Clinical Medical College/First Hospital of Shanxi Medical University, Taiyuan, China. ${ }^{4}$ Department of Clinical Psychology, Wuxi Mental Health Center, Nanjing Medical University, 156 Qianrong Road, Wuxi, Jiangsu Province 214151, China.

Received: 30 August 2018 Accepted: 27 March 2019

Published online: 15 April 2019

\section{References}

1. Lichtenstein P, Yip BH, Bjork C, Pawitan Y, Cannon TD, Sullivan PF, Hultman CM. Common genetic determinants of schizophrenia and bipolar disorder in Swedish families: a population-based study. Lancet. 2009;373(9659):234-9.

2. Sullivan PF, Kendler KS, Neale MC. Schizophrenia as a complex trait: evidence from a meta-analysis of twin studies. Arch Gen Psychiatry. 2003; 60(12):1187-92.

3. Havik B, Le Hellard S, Rietschel M, Lybaek H, Djurovic S, Mattheisen M, Muhleisen TW, Degenhardt F, Priebe L, Maier W, et al. The complement control-related genes CSMD1 and CSMD2 associate to schizophrenia. Biol Psychiatry. 2011;70(1):35-42.

4. Donohoe G, Walters J, Hargreaves A, Rose EJ, Morris DW, Fahey C, Bellini S, Cummins E, Giegling I, Hartmann AM, et al. Neuropsychological effects of the CSMD1 genome-wide associated schizophrenia risk variant rs 10503253. Genes Brain Behav. 2013;12(2):203-9.

5. Shimizu A, Asakawa S, Sasaki T, Yamazaki S, Yamagata H, Kudoh J, Minoshima S, Kondo I, Shimizu N. A novel giant gene CSMD3 encoding a protein with CUB and sushi multiple domains: a candidate gene for benign adult familial myoclonic epilepsy on human chromosome 8q23.3-q24.1. Biochem Biophys Res Commun. 2003:309(1):143-54.

6. Glancy M, Barnicoat A, Vijeratnam R, de Souza S, Gilmore J, Huang S, Maloney VK, Thomas NS, Bunyan DJ, Jackson A, et al. Transmitted duplication of 8p23.1-8p23.2 associated with speech delay, autism and learning difficulties. Eur J Hum Genet. 2009:17(1):37-43.

7. Cirulli ET, Kasperaviciute D, Attix DK, Need AC, Ge D, Gibson G, Goldstein DB. Common genetic variation and performance on standardized cognitive tests. Eur J Hum Genet. 2010;18(7):815-20.

8. Ripke S, Neale BM, Corvin A, Walters JTR, Farh K, Holmans PA, Lee P, BulikSullivan B, Collier DA, Huang $H$, et al. Biological insights from 108 schizophrenia-associated genetic loci. Nature. 2014;511:421-7.

9. Ripke S, Sanders AR, Kendler KS, Levinson DF, Sklar P, Holmans PA, Lin D, Duan J, Ophoff RA, Andreassen OA, et al. Genome-wide association study identifies five new schizophrenia loci. Nat Genet. 2011;43:969-76.

10. Cross-Disorder Group of the Psychiatric Genomics Consortium. Identification of risk loci with shared effects on five major psychiatric disorders: a genome-wide analysis. Lancet. 2013;381:1371-9.

11. Steen VM, Nepal C, Ersland KM, Holdhus R, Naevdal M, Ratvik SM, Skrede S, Havik B. Neuropsychological deficits in mice depleted of the schizophrenia susceptibility gene CSMD1. PLoS One. 2013;8(11):e79501.

12. Luykx JJ, Bakker SC, Lentjes E, Neeleman M, Strengman E, Mentink L, DeYoung J, de Jong S, Sul JH, Eskin E, et al. Genome-wide association study of monoamine metabolite levels in human cerebrospinal fluid. Mol Psychiatry. 2014;19(2):228-34.

13. Ohi $K$, Hashimoto $R$, Yamamori $H$, Yasuda $Y$, Fujimoto M, Umeda-Yano $S$, Fukunaga M, Watanabe $Y$, Iwase $M$, Kazui $H$, et al. The impact of the genome-wide supported variant in the cyclin M2 gene on gray matter morphology in schizophrenia. Behav Brain Funct. 2013;9:40.
14. Liu Y, Cheng Z, Wang J, Jin C, Yuan J, Wang G, Zhang F, Zhao X. No association between the rs10503253 polymorphism in the CSMD1 gene and schizophrenia in a Han Chinese population. Bmc Psychiatry. 2016;16(1).

15. Chana G, Bousman CA, Money TT, Gibbons A, Gillett P, Dean B, Everall IP. Biomarker investigations related to pathophysiological pathways in schizophrenia and psychosis. Front Cell Neurosci. 2013;7:95.

16. Kumarasinghe N, Beveridge NJ, Gardiner E, Scott RJ, Yasawardene S, Perera A, Mendis J, Suriyakumara K, Schall U, Tooney PA. Gene expression profiling in treatment-naive schizophrenia patients identifies abnormalities in biological pathways involving AKT1 that are corrected by antipsychotic medication. The International Journal of Neuro psychopharmacology. 2013; 16(07):1483-503

17. Sullivan PF, Fan C, Perou CM. Evaluating the comparability of gene expression in blood and brain. Am J Med Genet B Neuropsychiatr Genet. 2006;141B(3):261-8.

18. Kumarasinghe N, Tooney PA, Schall U. Finding the needle in the haystack: a review of microarray gene expression research into schizophrenia. Aust N Z J Psychiatry. 2012;46(7):598-610.

19. Frank A. Middleton,1 Karoly Mirnics, Joseph N. Pierri, David A. Lewis, Pat Levitt. Gene expression profiling reveals alterations of specific metabolic pathways in schizophrenia. J Neurosci 2002;22:2718-2729.

20. Crespo-Facorro B, Prieto C, Sainz J. Schizophrenia gene expression profile reverted to Normal levels by antipsychotics. Int J Neuropsychoph. 2015;18(4).

21. Xu Y, Yao SY, Wang G, Cheng Z, Jin C, Zhang K, Wang J, Yu H, Yue W, Zhang $F$, et al. Altered expression of mRNA profiles in blood of early-onset schizophrenia. Sci Rep. 2016:6:16767.

22. Choi KH, Higgs BW, Weis S, Song J, Llenos IC, Dulay JR, Yolken RH, Webster MJ. Effects of typical and atypical antipsychotic drugs on gene expression profiles in the liver of schizophrenia subjects. Bmc Psychiatry. 2009;9:57.

23. Shayegan DK, Stahl SM. Atypical antipsychotics: matching receptor profile to individual patient's clinical profile. CNS Spectr. 2004;9(10 Suppl 11):6-14

24. Agarwal V. Urinary incontinence with risperidone. J Clin Psychiatry. 2000; 61(3):219.

25. Sainz J, Prieto C, Ruso-Julve F, Crespo-Facorro B. Blood gene expression profile predicts response to antipsychotics. Front Mol Neurosci. 2018;11.

26. Kraus DM, Elliott GS, Chute H, Horan T, Pfenninger KH, Sanford SD, Foster S, Scully S, Welcher AA, Holers VM. CSMD1 is a novel multiple domain complement-regulatory protein highly expressed in the central nervous system and epithelial tissues. J Immunol. 2006;176(7):4419-30.

27. Escudero-Esparza A, Kalchishkova N, Kurbasic E, Jiang WG, Blom AM. The novel complement inhibitor human CUB and sushi multiple domains 1 (CSMD1) protein promotes factor I-mediated degradation of C4b and C3b and inhibits the membrane attack complex assembly. FASEB J. 2013;27(12):5083-93.

28. Shatz CJ. MHC class I: an unexpected role in neuronal plasticity. Neuron. 2009;64(1):40-5

29. Schafer DP, Stevens B. Synapse elimination during development and disease: immune molecules take Centre stage. Biochem Soc Trans. 2010; 38(2):476-81.

30. Schafer DP, Lehrman EK, Stevens B. The "quad-partite" synapse: microgliasynapse interactions in the developing and mature CNS. Glia. 2013;61(1):24-36.

31. Kwon E, Wang W, Tsai LH. Validation of schizophrenia-associated genes CSMD1, C10orf26, CACNA1C and TCF4 as miR-137 targets. Mol Psychiatry. 2013;18(1):11-2

Ready to submit your research? Choose BMC and benefit from

- fast, convenient online submission

- thorough peer review by experienced researchers in your field

- rapid publication on acceptance

- support for research data, including large and complex data types

- gold Open Access which fosters wider collaboration and increased citations

- maximum visibility for your research: over $100 \mathrm{M}$ website views per year

At $\mathrm{BMC}$, research is always in progress.

Learn more biomedcentral.com/submission 